\title{
Dubious Deductions from AGN Survey Data
}

\author{
Robert Antonucci \\ University of California, Physics Department, Santa Barbara, CA \\ 93106, USA
}

\begin{abstract}
The participants in this meeting are almost all carrying out the hard work of making many different types of AGN surveys. Since it's so much easier to criticize other people's work than to do actual work myself, I'll just present some demurs regarding recent papers drawing conclusions from various AGN survey data. In particular I'll mention some questionable interpretations of surveys of Seyfert 2 near-UV polarization; interpreting the results of searches for polarized broad $\mathrm{H}$-alpha lines in Seyfert 2s; testing the beam model for radio galaxies and quasars; testing the unification of Seyfert spectral types with a torus; and finally testing the energy sources for ULIRGs, especially those with LINER optical spectra. Only the polarized broad $\mathrm{H}-\alpha$ results are examined in detail here.
\end{abstract}

\section{Levels of Polarization of the Nuclear Light in Seyfert 2 Galaxies}

Koski (1978) showed that Seyfert 2s and Narrow Line Radio Galaxies generally have UV excesses relative to the SEDs for the Pop II stars which dominate the optical light in arcsec apertures. He showed the spectra could be parameterized approximately by a normal Pop II SED, plus a suitably normalized power law which dominates in the near-UV. The brightest powerful Seyfert 2 nucleus, that of NGC1068, can indeed be fit quite well that way (Miller and Antonucci 1983, McLean et al 1983, Antonucci et al. 1994), and furthermore, the powerlaw component has a wavelength-independent polarization of $\sim 16 \%$ in arcsec apertures. It is crucial to note that the broad permitted lines show the same percent $\mathrm{P}$ as the power-law continuum component accounting for the "UV excess". This strongly suggests that the scattering paths of the broad lines and the power law are very similar geometrically. Finally, the permitted lines have normal equivalent width in polarized flux. This confirms that the hidden source is simply a normal Seyfert 1 nucleus, whose light is scattered into the line of sight.

The polarization angles of NGC1068 and other Seyfert 2s lie perpendicular to the radio axes, so that the photons' last flights before scattering into the line of sight were along the axes. Thus the equatorial directions must be opaque, and the torus model was born. In principle the polar scattering could result from intrinsically anisotropic emission, but the anisotropy would need to be the same for the broad emission lines as for the power law, which would be difficult to arrange by some mechanism other than shadowing. In fact if these 
two components differ substantially in their isotropy in any significant number of objects, we'd expect to see one component without much of the other fairly frequently in Type 1 objects. The broad line equivalent width dispersion would then be very large and this is not seen.

It was evident from the beginning of the era of high SNR spectropolarimetry that most other objects behave somewhat differently (Antonucci 1984 and Tran et al. 1995 on 3C234; Miller and Goodrich 1990; Tran 1995). It's quite general that the broad line equivalent widths are normal in the polarized flux spectra of narrow line objects, that is, they match those of broad line objects. Thus the basic picture is the same as for NGC1068: a normal broad line nucleus is seen in reflection. But in general the UV "featureless continuum" has a polarization much lower than that of the broad emission lines (Kay 1994). Thus there is another, unpolarized UV continuum component, now known to derive from hot stars in many cases (Heckman et al. 1995, Gonzalez-Delgado et al. 1998). But the key from the unified model point of view is that the broad lines are highly polarized (at 16\%, NGC1068 has about the lowest $\mathrm{P}$ ), so that the scattering is in one or two polar cones.

The point here is that several authors have taken the percent $P$ from the UV continuum--which is often low-and rejected polar scattering on that basis. For example, see the key role of this erroneous argument in the discussion of Malkan et al. $1998 .^{1}$

\section{Polarized Broad H- $\alpha$ and the Generality of Seyfert Unification}

Since the early days of the geometrical unified models of various kinds, attempts have been made to assess the generality of the models. For example, Tran (2001) has recently discussed whether or not all Seyfert 2s have hidden Type 1 nuclei of any significant luminosity. It's usually difficult to prove that something is not present, and people haven't yet converged on the answer to this. ${ }^{2}$

Tran (2001) has given a variety of arguments and concludes that there are in fact many "real" Seyfert 2 galaxies without hidden Type 1 nuclei. His paper may be correct but I want to take this opportunity to review some places where

\footnotetext{
${ }^{1}$ The way to measure the broad line polarization, and hence the polarization of the scattered light alone, is to divide the polarized flux by the total flux. However, it is generally impossible to see the line clearly in total flux, so one just derives high lower limits to the broad line polarization in most cases. Tran (1995) claimed to measure the broad lines in total flux in some objects. However, M. Kishimoto and I looked carefully at the case of Mrk 477, in preparing a paper on polarization imaging. We examined the total-flux plots, overlaying the permitted Ba lines on various forbidden lines; we saw little or no evidence for broad wings in the total flux. We thus placed an upper limit on the total flux of the broad components, leading to a conservative lower limit on broad $\mathrm{H}-\alpha$ and $\mathrm{H}-\beta$ polarization of $10 \%$. Thus we disagree with the intrinsic value of only $2-3 \%$ quoted in Tran 1995 . We used the same data.

${ }^{2}$ One way to do it is to see whether there's any significant "waste heat"-dust reradiation from the matter obscuring the putative hidden nucleus. D. Whysong and I are studying $3 \mathrm{C}$ radio galaxies in the mid-IR with this in mind, and have shown, for example, that M87 can have no such hidden nucleus at any remotely relevant luminosity level: astro-ph 0106381. Also, Meisenheimer et al. (2001) present a very good study of ISO data, and reach a similar conclusion that many lower-luminosity radio galaxies lack significant waste heat.
} 
his arguments seem quite uncertain - most of these are already discussed in his paper.

Tran (2001) presents spectropolarimetric data on two Seyfert 2 samples: the $12 \mu$-selected sample of Rush et al. 1993, and the CfA sample of Huchra and Burg 1992. The main finding seems to be that those with detected hidden broad emission line regions ("HBLRs") have higher radio power relative to the far-IR power than the non-HBLRs on average, and that they also have warmer $25 \mu-60 \mu$ colors on average. These differences are taken to show that the two types (HBLRs vs. non-HBLRs ${ }^{3}$ ) of Seyfert 2 are intrinsically different, and that in particular there is a large subset with no hidden Type 1 nucleus.

I've often preached about the great improvement in interpretability of tests of this type when the samples are selected by a property which is thought to be fairly isotropic, such as far-IR, and $\mathrm{H}$. Schmitt explores this in detail in his article in this volume. (See also Schmitt et al. 2001.) That's certainly not the case with the $12 \mu$ sample, since the very high column densities of the tori result in substantial optical depths at that wavelength, though this sample is much better in this regard than those based on say, UV excess. Aside from all theoretical considerations, and aside from reference to the X-ray columns, it seems obvious that the $12 \mu$ emission is quite anisotropic simply because the HBLR Seyfert $2 \mathrm{~s}$ have much steeper mid-IR spectra than bare Type 1 nuclei (see e.g., Edelson \& Malkan 1986). This wouldn't be the case if the mid-IR were emitted isotropically. The X-ray columns reinforce this: they are much larger in the Type $2 \mathrm{~s}$ of all kinds than in the Type 1s, and this huge extra column density is probably dusty molecular gas. That last point is confirmed semiquantitatively at least from molecular line maps. ${ }^{4}$ See for example Planesas et al. 1991. Thus I can't agree with the characterization of the $12 \mu$ sample as complete and unbiased with respect to orientation.

For a few important related fine points of Tran's paper, he asserts that the contradictory conclusions of Heisler et al. 1997 may be due to sample "incompleteness" of the latter. As discussed below and in Schmitt's contribution to these proceedings, I believe the selection of the latter is in fact better, based largely on the far-IR which is thought to be relatively isotropic. The Heisler paper was recently updated as Lumsden et al. 2001, and the interpretation given there is more consistent with Tran's interpretation.

Other caveats about Tran's paper:

1) The polarimetry data come from Lick, Palomar and Keck Observatories. No indications are made that the observations are of uniform depth, and no upper limits are given for broad $\mathrm{H}$-alpha for the putative non-HBLRs. It would not be simple to rectify this situation. However, I already see from a footnote

\footnotetext{
${ }^{3}$ Note that "non-HBLR" in Seyfert $2 \mathrm{~s}$ means there is no BLR present at all, since the Seyfert 2 classification precludes a visible BLR.

${ }^{4}$ The X-ray columns in Type 1 nuclei, while much smaller in general, are still usually larger than those expected from the optical reddening/extinction estimates, for Galactic dust size distributions and dust/gas ratios (Maiolino et al. 2001 and references therein). It's possible that this gas contains only large grains, or perhaps is dust-free, but it's quite a different issue from the enormous excess columns of Type $2 \mathrm{~s}$ relative to Type 1s.
} 
to Moran et al. 2001 that two of Tran's "non-HBLR" objects do show the broad $H-\alpha$ in polarized flux with better data.

2) The warm mid-IR average colors of the HBLR objects have been discussed before (e.g. Heisler et al. 1997, Lumsden et al. 2001; Alexander 2001, and references therein; see also Miller and Goodrich 1990), with arguments as to whether the warmer colors might derive from lower inclinations as expected for tori theoretically. That could explain the lack of polarized broad $\mathrm{H} \alpha$ if the objects with the highest inclinations have partially obscured scattering regions. There is also evidence given in these papers that at least part of the effect (the correlation between detectability of a scattered BLR and mid-IR "warmth") is a contrast issue related to the relative dominance of the AGN in a particular object, and this seems natural and unavoidable too.

Tran argues (following Alexander 2001) that the cooler mid-IR spectra of the non-HBLRs cannot be due to higher extinction to the warm regions (and thus presumably to torus inclination) because the X-ray columns for the samples under consideration are statistically indistinguishable. To me that's suggestive at best because of the sample mismatches, and more importantly, because it's a purely qualitative argument. No theory predicts the magnitude of the expected difference between the columns of the HBLRs and non-HBLRs if the difference is due to inclination. One would need to have such a robust prediction to compare with any observational upper limit on the statistical column density difference, in order to prove a discrepancy.

3) A point that I find at least strongly suggestive (given the problematic selection criteria), is that the HBLR Seyfert $2 \mathrm{~s}$ have a larger ratio of radio/far-IR than the others. ${ }^{5}$ This seems to be a modest effect though (see Tran's Fig. 1; no statistical significance is given in the paper, but a significance of $0.8 \%$ is given by a K-S test: H.T., pc). If it is accepted to be statistically significant for the two populations, a next step would be to examine whether free-free absorption in the radio would be significant for the objects of highest inclination. Many recent papers on Seyfert radio properties have concluded free-free optical depths can be substantial in the $\mathrm{cm}$ region (e.g., Gallimore et al. 1997, Ulvestad 1999, and several others). Also a starburst component might have a different radio/far-IR ratio. But a different starburst contribution doesn't constitute strong evidence against a hidden BLR.

Tran and also Thean et al. 2001 find that the absolute radio luminosities of the HBLR Seyfert $2 \mathrm{~s}$ are significantly greater than those of the non-HLBRs for the $12 \mu$ sample. However, with imperfect selection criteria, I'm more comfortable with comparing ratios of nearly isotropic properties such as discussed in the previous paragraph.

Also a general caveat should be kept in mind. The fact that a statistical difference in the populations of the putative types of 2 may exist is only suggestive of a qualitative difference in the physics. If all have hidden BLRs, the

\footnotetext{
${ }^{5}$ The paper also cites Moran et al. 1992 as providing evidence for intrinsically greater absolute radio luminosities in HBLR Seyfert $2 \mathrm{~s}$; that result was subject to severe selection effects, and was essentially retracted in Moran et al. 2000. Certainly given the anisotropic selection criteria for the Tran objects, I'd hesitate to make much of the radio power difference in the Tran paper.
} 
two types would necessarily differ in average torus covering factor, so needn't be identical in other intrinsic properties.

4) The point is made that the [OIII] $5007 / \mathrm{H} \beta$ ratio median is $9.9 \pm 1.3$ for the HBLR nuclei but only $6.8 \pm 1.5$ for the non-HBLRs. This brings up the vexing question of definitions for Seyfert 2. Well known examples of bright Seyfert 2s have ratios near 10. Half of the "non-HBLR Seyfert $2 \mathrm{~s}$ " as used in the paper have ratios less than 6.8. The ratios aren't tabulated in the paper, but I'd interpret this as meaning that many so-called non-HBLRs are composites, with important LINER or starburst contributions to many of them. Certainly these components will reduce the integrated dust temperatures in the sense observed. But again that doesn't constitute evidence against a hidden BLR commensurate with the amount of high-ionization narrow line gas present.

5) Tran asserts that the non-HBLR Seyfert 2 s simply have no hidden BLR. But the BLR is always accompanied by a "power-law" continuum, and this continuum is the only viable explanation for some of the narrow line ratios. Thus the question arises: how are the non-HBLR narrow line regions ionized? He speculates that the latter are "dominated by other nuclear and circumnuclear processes such as starbursts." This seems consistent with the statement that they have lower excitation, but also seems to reduce the question to semantics. No one doubts that there are objects dominated by e.g. starbursts, which thus have different narrow line ratios than Seyferts... it's just that the starbursts are relatively more important in some objects than in others, and the excitation is a measure of that. Again I don't see any implication that there's no hidden BLR commensurate with the requirements for narrow line ionization level in a particular object. ${ }^{6}$

6) The paper of Pappa et al. (2001) is cited as showing the existence of low intrinsic X-ray absorption in two Seyfert 2 s of very low luminosity. Many Seyfert 2's are Compton thick and thus show no absorption turnover at low $\mathrm{X}$-ray energies, but for these two, other arguments are given that suggest that this isn't likely. As Pappa et al. point out, the lack of an observed BLR could be intrinsic, or else it could be due to a dusty warm absorber as documented in several more luminous objects. I'm not aware that either of these two, NGC3147 or NGC 4698, has been checked for broad polarized $\mathrm{H} \alpha$, but that would certainly be worthwhile.

My personal conclusion from all this is that the existence of objects without the Big Blue Bump and accompanying broad emission lines-commensurate with the luminosity of highly excited narrow line gas-has not been shown robustly. This is actually pretty similar to Tran's conclusion that "it is the strength of the AGN engine that seems to be the dominant factor in determining the visibility of the HBLR." Undoubtedly if the nuclear continuum and BLR strength can be turned down in a particular object, everything else being the same, the

\footnotetext{
${ }^{6}$ Nuclear activity seems manifest mainly as the Big Blue Bump and the resulting broad and narrow emission lines in radio quiet AGN. There is no question that some radio galaxies lack significant visible or hidden $\mathrm{BBBs}$, based on the mid-IR argument mentioned in Footnote 2, but they are still AGN because of the radio activity. But what AGN activity exists in a nonHBLR non-radio-loud Seyfert 2? In finance, the analogy would be a bond which is a "zero coupon perpetuity," which makes no interest payments, and never pays back the principle. At that point it's not much of a bond, and a non-HBLR Seyfert 2 might be similarly ill-defined.
} 
scattered light signal would be harder to see. The question is, does anything happen qualitatively at low AGN luminosities? Do these components decrease faster than say the strength of the high-excitation narrow line gas? It seems to me we're as far as ever from answering that question.

\section{Testing the Generality of Seyfert Unification with Isotropic Prop- erties}

It would be great to have a dollar for every paper that concluded that Seyfert $1 \mathrm{~s}$ and $2 \mathrm{~s}$ are intrinsically different by showing that samples differ in some way, without selecting the sample by an isotropic property. No unified model says that any old batch of objects of one type is equivalent to any old batch of objects of another type. As an extreme example, with UV selection, an object whose UV excess is just scattered light must be much more powerful intrinsically than one whose UV excess is seen directly. For objects like NGC1068, only a percent or so of the nuclear UV is scattered into the line of sight, so Seyfert $2 \mathrm{~s}$ found in this way come from five magnitudes higher on the luminosity function than Seyfert 1 s found this way. No wonder they have more CO, L(far-IR), radio emission, etc, etc! I'll just mention one recent example. The Malkan et al 1998 paper, based on the Seyferts in the HST archives, concludes that Seyfert 2s are more likely to have nuclear dust structures. According to the verbal contribution of Maiolino et al. to the Guillermo Haro Workshop held at UNAM in 2000, and in a pc, this effect disappears if the Seyfert types are matched for [OIII] luminosity. (Other demurs regarding Malkan et al. 1998 can be found in Antonucci 1999a.)

A must-read paper in this context is Keel et al. 1994, which analyzes properties of Seyferts selected by $60 \mu$ flux and $25 \mu-60 \mu$ color. That sample should be pretty good-maybe the best that is available right now. Several old saws about Seyfert 2s having greater narrow line luminosities, ratios of narrow line luminosities to radio, etc, are disproven there. Not everything in Keel et al. can be easily explained by orientation though!

H. Schmitt is leading a major program of study of the sample from the Keel et al. paper, and he gives a report in these proceedings. (See also Schmitt et al. 2001.) He shows that several claimed differences between Seyfert $1 \mathrm{~s}$ and $2 \mathrm{~s}$ go away with this type of selection. To make the sample even better, we hope to add in the Seyferts dropped from the Keel et al list because of the $25 \mu-60 \mu$ color criterion. The result should be quite good.

There is a crucial limitation of this type of test, however. As emphasized in the past by e.g., A. Lawrence, there must be a range of covering factors for the dusty tori, and so those classified as Type 2 must have higher average covering factors. Therefore as populations, the 1s and $2 \mathrm{~s}$ must be intrinsically different in their statistical properties at some level. This has no direct implication that some objects lack hidden BLRs!

To reiterate, even a perfect study, using isotropic selection and real upper limits, is expected to show differences between $1 \mathrm{~s}$ and $2 \mathrm{~s}$ in their intrinsic statistical properties. One could only confidently expect broad overlap in the properties of the $1 \mathrm{~s}$ vs. the $2 \mathrm{~s}$ even if every 2 has a hidden Type 1 nucleus. Such a difference would by itself have no direct implication that some of the Type $2 \mathrm{~s}$ lack a BLR. 


\section{Testing the Beam Model for Radio Galaxies and Quasars with Isotropic Properties}

This is an old issue, pretty much settled long ago I think. It still carries a good lesson, very closely analogous to that of the previous section. Blandford and Rees (1978) and Blandford and Konigl (1979) proposed that superluminal radio sources were simply normal double sources seen from very low inclinations, i.e., seen from along the axes of the radio jets. Early tests of the idea using the associated radio projected linear sizes (Browne ${ }^{7}$ et al. 1982) and the narrow emission lines (Heckman 1983) found major discrepancies with it. However the double-lobed objects used in the comparison samples to the superluminals came from orders of magnitude higher on the luminosity function, because they satisfied survey flux limits with their nearly isotropic diffuse radio emission, whereas the superluminals satisfied survey flux limits because of their beamed core emission. It was exactly like the Seyfert $1 / 2$ sample problems referred to in the last section. This was discussed in detail in Antonucci and Ulvestad 1985.

\section{Testing the Energy Sources for Ultraluminous Infrared Galaxies with Mid-IR Spectroscopy}

As for the previous section, I'll give just a brief sketch of this important issue, with a reference to a recent fuller discussion: Antonucci 2001.

The issue is the energy sources in Ultraluminous Infrared Galaxies, and whether recent ISO mid-IR spectral surveys provide breakthroughs in this area.

A large subset of the ULIRGs have properties exactly as expected for ultraluminous "Quasar 2s," that is to say, powerful high-ionization narrow line emission, powerful mid-far infrared emission, no optical point sources, but instead diffuse "mirror" regions revealing light from a hidden quasar. Note that the optical continuum is not expected to scale with nuclear luminosity because in low luminosity Seyfert 2s, it's almost always dominated by the underlying old stellar population.

The narrow emission lines have much lower equivalents widths in quasars than in Seyfert 1s, so their luminosity doesn't scale proportionately to the nuclear optical/UV either (e.g., Boroson \& Green 1992, and Wills et al. 1993). It's a big effect.

As with nearby Seyfert 2s, it's difficult to be very precise about any starburst contribution to ULIRG energetics, but some well-studied cases reveal that it is often substantial. However, it is almost universally true that the objects characterized by Seyfert-2 like narrow line ratios in the optical show the same type of emission line spectrum in the mid-IR. Thus, the latter spectral region just reveals the same emission component as the optical for this group of ULIRGs.

At lower luminosities especially, many ULIRGs have starburst optical spectra, and also starburst mid-IR spectra. Again the mid-IR observations reveal just the same emission region as the optical, at least in a qualitative sense.

\footnotetext{
${ }^{7}$ Browne was, however, an early advocate for matching diffuse radio power.
} 
Where the mid-IR data show something new is among the many ULIRGs with LINER optical spectra: in almost all of these cases the mid-IR spectra show lines from HII regions (e.g., Lutz et al. 1999)! This is interpreted most simply (and in the published papers, essentially solely) as proving that the true nuclei, and by implication the dominant energy sources, are compact starbursts. However, in general the star formation uncovered this way cannot be shown to dominate (or otherwise) the galaxy energetics because there is no accurate conversion from any particular starburst spectral feature to the bolometric luminosity of the associated starburst population.

Thus the ISO data show the presence of substantial but poorly determined starburst luminosity somewhere in the galaxies, generally at the obscuration depth penetrated by light of these wavelengths. Unfortunately, obscured AGN are known to possess column densities of $\gtrsim 10^{24} \mathrm{~cm}^{-2}$ in most cases, where $A(V) \gtrsim 1000$ and the mid-IR from the nucleus is quite obscured. There is a moderate range of parameter space such that the mid-IR can't penetrate the gas/dust columns, but the hard X-rays can. Above $>10^{25} \mathrm{~cm}^{-2}$ or so however, the dusty gas becomes "Compton thick" and even the hard X-rays are blocked.

The point is that recently many ULIRGs classified as LINERS in the optical and starbursts in the mid-IR have since revealed powerful $\sim 10 \mathrm{keV}$ X-ray sources, often with luminosities suggestive of hidden AGN which are capable of providing the entire observed bolometric power. In other LINER ULIRGs a hidden powerful AGN is revealed by spectropolarimetry, though with that technique we have no good way of estimating the contribution of the AGN to the bolometric luminosity. These cases show that published conclusions that $>50 \%$ of the energy in a ULIRG derives from a hidden starburst are invalid. The same applies to the SCUBA sources, which were placed directly on the "Madau" diagram of stellar luminosity density evolution, without the slightest evidence that they're powered by stars!

The details and references are given in the review article cited at the top of this section (as well as in papers by experts such as Sanders and Mirabel, and Veilleux and several others). I just mention two cases here because they are really amusing to me. The well-studied ULIRG NGC6240 is a LINER in the optical, but a starburst in the mid-IR. In fact it's a "template" starburst in the mid-IR according to Genzel et al. 1998. The Genzel team claims to have shown that this and many other examples are starburst- dominated on this basis. Only trouble is, NGC6240 and many others have powerful AGN X-ray sources coming directly through the obscuring matter at $10 \mathrm{keV}$ (Vignati et al. 1999).

I don't know of any starburst spectral feature that can be accurately used to determine the starburst bolometric luminosity, so the starburst features can only say that there is such a component, and put a relatively low minimum value on its luminosity. The situation is a little better for AGN in that the X-ray luminosity is empirically a pretty good ( factor of 3 ?) estimator of the bolometric value, based on the unobscured cases. Vignati et al. (1999) point out that that relation indicates that the bolometric luminosity of the hidden AGN in NGC6240 is consistent with the entire observed bolometric luminosity. Conservatively, I conclude from that that the AGN makes a significant contribution, though it's not certain that it's $>50 \%$. 
The situation for NGC4945 is similar. Spoon et al. (2000) have analyzed the mid-IR spectrum finding that the starburst "may well power the entire bolometric luminosity, ... [but] are also consistent with an up to $50 \%$ contribution from an embedded AGN." Immediately afterward, Madejski et al. (2000) published a spectacular wide-band SED showing the few times $10^{24} \mathrm{X}$-ray column, and deriving the unabsorbed nuclear X-ray luminosity alone at $\sim 10^{43} \mathrm{erg} / \mathrm{s}, \mathrm{a}$ substantial fraction of that in the mid-far-IR, and indicative of a bolometric luminosity consistent with that observed. It is very important to note that there are no indications of activity in NGC4945 other than the hard X-ray source. This means that active galaxies can look perfectly normal at other wavelengths! They do not need to show even the LINER-like emission lines!

\section{Acknowledgements}

For comments on an earlier draft I thank L. Kay, T. Heckman, B. Wills, M. Kishimoto, H. Schmitt, J. Ulvestad, and R. Barvainis. Detailed comments from B. Wills led to many changes. Support came from NSF grants NSF AST9617160 and NSF AST00-98719.

\section{References}

Alexander, D. M. 2001, MNRAS, 320, L15

Antonucci, R. R. J. 1984, ApJ, 278, 499

Antonucci, R. R. J. \& Ulvestad 1985, ApJ, 294, 158A

Antonucci, R., Hurt, T., \& Miller, J. 1994, ApJ, 430, 210

Antonucci, R. 1999, ASP Conf. Ser. 161: High Energy Processes in Accreting Black Holes, 193 -also known as astro-ph/9810067

Antonucci, R 2001 - astro-ph /0103048 - to be pub in AGN Surveys, Proceedings of IAU Colloquium 184. Edited by R.F. Green, E.Ye. Khachikian, and D.B. Sanders. Publisher: ASP, Dates: June 18-22, 2001, Location: Byurakan, Armenia

Blandford, R. D. \& Rees, M. J. 1978, Pittsburgh Conference on BL Lac Objects, Pittsburgh, Pa., April 24-26, 1978, Proceedings. (A79-30026 11-90)

Pittsburgh, Pa., University of Pittsburgh, 1978, p. 328-341

Blandford, R. D. \& Konigl, A. 1979, ApJ, 232, 34

Boroson, T. A. \& Green, R. F. 1992, ApJS, 80, 109

Browne, I. W. A., Clark, R. R., Moore, P. K., Muxlow, T. W. B., Wilkinson, P. N., Cohen, M. H., \& Porcas, R. W. 1982, Nature, 299, 788

Edelson, R. A. \& Malkan, M. A. 1986, ApJ, 308, 59

Gallimore, J. F., Baum, S. A., \& O'Dea, C. P. 1997, Nature, 388, 852

Genzel, R. et al. 1998, ApJ, 498, 579

González Delgado, R. M., Heckman, T., Leitherer, C., Meurer, G., Krolik, J., Wilson, A. S., Kinney, A., \& Koratkar, A. 1998, ApJ, 505, 174

Heckman, T. M. 1983, ApJ, 271, L5

Heckman, T. et al. 1995, ApJ, 452, 549 
Heisler, C. A., Lumsden, S. L., \& Bailey, J. A. 1997, Nature, 385, 700

Huchra, J. \& Burg, R. 1992, ApJ, 393, 90

Kay, L. E. 1994, ApJ, 430, 196

Keel, W. C., de Grijp, M. H. K., Miley, G. K., \& Zheng, W. 1994, A\&A, 283, 791

Koski, A. T. 1978, ApJ, 223, 56

S.L. Lumsden (1,2), C.A. Heisler (3), J.A. Bailey (2), J.H. Hough (4), S. Young, MNRAS, in press. Also known as astro-ph/0106263.

Lutz, D., Veilleux, S., \& Genzel, R. 1999, ApJ, 517, L13

Maiolino, R., Marconi, A., \& Oliva, E. 2001, A\&A, 365, 37

Malkan, M. A., Gorjian, V., \& Tam, R. 1998, ApJS, 117, 25

McLean, I. S., Aspin, C., Heathcote, S. R., \& McCaughrean, M. J. 1983, Nature, 304,609

Meisenheimer, K., Haas, M., Müller, S. A. H., Chini, R., Klaas, U., \& Lemke, D. 2001, A\&A, 372, 719

Miller, J. S. \& Antonucci, R. R. J. 1983, ApJ, 271, L7

Miller, J. S. \& Goodrich, R. W. 1990, ApJ, 355, 456

Moran, E. C., Halpern, J. P., Bothun, G. D., \& Becker, R. H. 1992, AJ, 104, 990

Moran, E. C., Barth, A. J., Kay, L. E., \& Filippenko, A. V. 2000, ApJ, 540, L73

Moran, E. C., Kay, L. E., Davis, M., Filippenko, A. V., \& Barth, A. J. 2001, ApJ, 556, L75

Pappa, A., Georgantopoulos, I. Stewart, G.C., Zezas, A.L. astro-ph/0104061, to be published in MNRAS

Planesas, P., Scoville, N., \& Myers, S. T. 1991, ApJ, 369, 364

Rush, B., Malkan, M. A., \& Spinoglio, L. 1993, ApJS, 89, 1

Schmitt, H. R., Antonucci, R. R. J., Ulvestad, J. S., Kinney, A. L., Clarke, C. J., \& Pringle, J. E. 2001, ApJ, 555, 663

Spoon, H. W. W., Koornneef, J., Moorwood, A. F. M., Lutz, D., \& Tielens, A. G. G. M. 2000, A\&A, 357,898

Tran, H. D. 1995, ApJ, 440, 578

Tran, H. D. 2001, ApJ, 554, L19

Ulvestad, J. S., Wrobel, J. M., Roy, A. L., Wilson, A. S., Falcke, H., \& Krichbaum, T. P. 1999, ApJ, 517, L81

Vignati, P. et al. 1999, A\&A, 349, L57 